\title{
„Neue Perspektiven und Orientierungen" - Anmerkungen zum Kongress des Lateinamerikanischen Germanistenverbandes in Buenos Aires 2017
}

Johannes Köck

Von 27. November bis 1. Dezember 2017 fand in Buenos Aires, Argentinien, der 16. Kongress des Lateinamerikanischen Germanistenverbandes (ALEG) unter dem Titel Germanistik in Lateinamerika: Neuorientierungen - Neue Perspektiven ${ }^{1}$ statt. Da der ALEG ${ }^{2}$ als bedeutendster Fachkongress der lateinamerikanischen Germanistik gilt ${ }^{3}$ und zudem sechs Vortragende von tschechischen Universitäten vertreten waren, sollen nachfolgende einige kurze Einblicke und Anmerkungen aus der involvierten Perspektive eines Teilnehmenden geschildert werden. Austragungsort des ALEG war das Instituto de Enseñanza Superior en Lenguas Vivas, eine Sprachhochschule im Kolonialstil, die sich im Zentrum der argentinischen Hauptstadt befindet. Aufgrund der großen zu erwartenden Anzahl an Teilnehmer_innen erfolgte bereits im Vorfeld der Appell der Veranstalter_innen, auf den Zeitrahmen von maximal 20 Minuten pro Vortrag zu achten. Pro Mesa (la Mesa Spanisch für Tisch) referierten je vier Vortragende zu einem spezifischen Thema. Diese Mesas (Themenblöcke) dauerten insgesamt ca. 90 Minuten, da im Anschluss an alle Vorträge eine kurze plenare Fragerunde und Debatte stand, welche von einem/einer Coordinador(a)/Moderator_in koordiniert wurde. Um einen kurzen thematischen Überblick zu gewähren sollen nachfolgend die geplanten Sektionen des ALEG aufgelistet werden:

a) Deutschsprachige Literatur

b) Komparatistik

c) Literatur in Beziehung zu anderen Medien, Künsten und Diskursen

1 XVI Congreso de la Asociación Latinoamericana de Estudios Germanísticos (ALEG) Germanistica en Latinoamérica: nuevas orientaciones - nuevas perspectivas.

2 ALEG wird nachfolgend als Sigle für den Kongress des Lateinamerikanischen Germanistenverbandes verwendet.

3 Der nur vierjährlich an wechselnden Orten stattfindende Kongress wird unter der Mithilfe vieler Gremien und Verbände organisiert. Eine dezidierte Aufstellung würde den Rahmen dieses Kurzbeitrages sprengen. Nähere Infos waren unter der Kongresshomepage http://www.aleg2017.com/ und sind leider nicht mehr verfügbar. 

d) Literaturtheorie und Ästhetik
e) Übersetzungswissenschaft
f) Linguistik
g) Didaktik der deutschen Sprache
h) Emigrantenliteratur -Exilliteratur
i) Geschichte der Germanistik

Die ursprünglich angedachten Sektionen wurden vor Beginn des Kongresses noch ergänzt und ausdifferenziert. So gab es etwa gleich drei verschiedene Sektionen zur zeitgenössischen Literatur und verschiedenste Untersektionen/Mesas zu den jeweiligen übergeordneten Themenblöcken. Obwohl der 29. November zur freien Verfügung stand und für unterschiedliche Stadtspaziergänge genutzt wurde, und somit „nur“ an vier Tagen Vorträge stattfanden, bot der ALEG ein außergewöhnlich umfangreiches Fachprogramm. Umrahmt wurde dieses von einer einführenden musikalischen Eröffnung samt Grußworten und einer ebensolchen feierlichen Abschlusskundgebung. Die Vielfalt und Größe des Kongresses wird vielleicht durch die nachfolgenden Zahlen illustriert: 271 Vortragende in 62 Mesas, fünf Plenarvorträge, Panels, Verlagspräsentationen und Podiumsgespräche zeugen von den enormen Ausmaßen des größten Germanistikkongresses in Lateinamerika. Die Vielzahl der Beiträge führte auch zu einer großen thematischen Bandbreite, was sich bereits durch einen Blick auf die Vorträge der Teilnehmenden von tschechischen Universitäten verdeutlichen lässt. Insgesamt sechs Vortragende referierten zu Themen der Sektionen Deutsch als Fremdsprache (3TN), zeitgenössische deutschsprachige Literatur, Mediensprache und Pragmatik (je 1TN). Die sechs Dozent_innen aus der Tschechischen Republik sind in Ostrava (1), Ústí nad Labem (2) und Brno (3) tätig und hielten folgende Präsentationen:

Prof. Dr. Lenka Vaňková (Universität Ostrava): Fachwort in der deutschen Tagespresse. Sektion Mediensprache.

Doz. Dr. Renata Cornejo (Universität J. E. Purkyně Ústí nad Labem): Peripherie oder Zentrum? Interkulturelle Literatur, dargestellt am Beispiel von Autorinnen tschechischer Herkunft. Sektion Zeitgenössische deutsche Literatur I.

Doz. Dr. Hana Bergerová (Universität J. E. Purkyně Ústí nad Labem): 30 Jahre Phraseodidaktik des Deutschen als Fremdsprache: Ein Rück- und Ausblick. Sektion Linguistische Ansätze für den DaF-Unterricht.

Doz. Dr. Jiřina Malá (Masaryk-Universität Brno): Phraseologismen als Ausdrucksmittel von Emotionen. Sektion Pragmatik.

Johannes Benjamin Köck (DAAD-Lektor an der Masaryk-Universität Brno): Wider die Konstruktion von Fremdheit - Das Potential literarischer Texte im DaF-Unterricht. Sektion Literatur- und Dramenpädagogik im DaF-Unterricht.

Prof. Dr. Věra Janíková (Masaryk-Universität Brno): Linguistic landscapes im DaF-Unterricht. Sektion Linguistische Ansätze für den DaF-Unterricht.

Das große thematische Spektrum und die schiere Dimension des ALEG erwiesen sich naturgemäß nicht nur als Vorteil. Aufgrund der vielen Teilnehmenden und Sektionen 

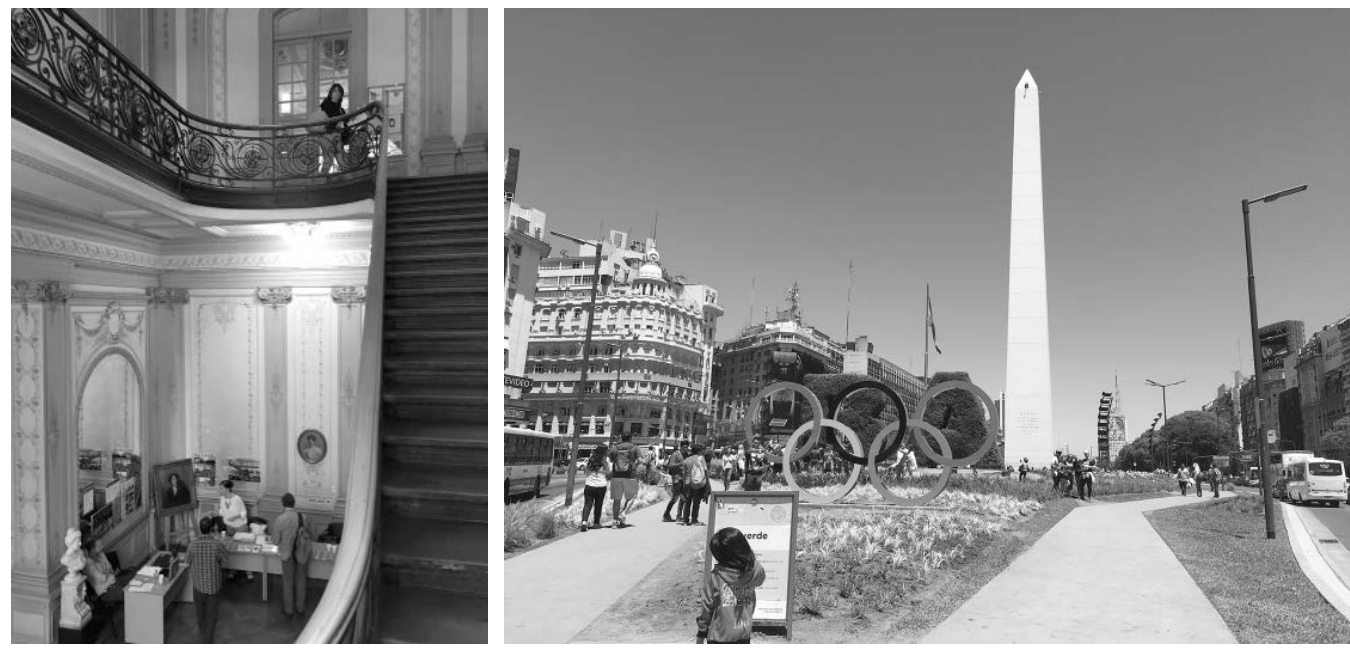

war es schwer sozial zu interagieren und internationale Netzwerke zu knüpfen - trotz des Ausflugtages. Mehr kurze Pausen zwischen den Sektionen, in denen die Teilnehmenden ins Gespräch kommen können, wären hier hilfreich gewesen. Als weiteres Problem stellte sich der Vortragsmodus dar: Vorträge in den drei offiziellen Sprachen des Lateinamerikanischen Germanistikverbandes Deutsch, Spanisch, Portugiesisch, waren möglich, wobei im Falle von Vorträgen auf Spanisch und Portugiesisch gebeten wurde, eine deutsche Fassung oder schriftliche Zusammenfassung zu bieten. Diese Anmerkungen waren aber wenig verbindlich, konkrete Hinweise in welcher Form eine mehrsprachige Präsentation möglich sein könnte (etwa graphisch hervorgehobene Schlüsselwörter auf jeder Folie, oder Hinweise zum konsekutiven Übersetzen/Dolmetschen) wären hilfreich gewesen. Die Folge war leider, dass einige Beiträge nur auf Spanisch oder Portugiesisch gehalten wurden, wodurch viele Anwesende in keinster Weise partizipieren konnten. (Selbst)kritisch muss jedoch angemerkt werden, dass wir Teilnehmende es versäumt haben, eigenorganisiert einen Modus zu finden, in dem wir einander konsekutiv dolmetschen. Vielleicht wäre es für den nächsten - voraussichtlich in Cuba stattfindenden ALEG - eine Möglichkeit, Studierende der Translationswissenschaft aktiv mit einzubeziehen?

Als Anregung für den germanistischen Diskurs in Tschechien bleibt der Wunsch, dass „Neue Perspektiven und Orientierungen“ noch mehr als bisher instituts- und städteübergreifend entworfen und diskutiert werden.

Zudem soll es abschließend trotz der mitunter kritischen Anmerkungen nicht versäumt werden, den Veranstaltern für die Organisation eines insgesamt sehr gelungenen und anregenden Kongresses in einer faszinierenden Stadt zu danken ${ }^{4}$ : Agradezco a los organizadores del XVI Congreso de la Asociación Latinoamericana de Estudios Germanísticos - „GRACIAS“!

4 Ich danke den Organisatoren des 16. Kongresses des Lateinamerikanischen Germanistenverbandes „Danke“. 
Johannes Benjamin Köck / koeck@mail.muni.cz

Masarykova univerzita, Filozofická fakulta, Ústav germanistiky, nordistiky a nederlandistiky Arna Nováka 1, 60200 Brno, CZ 